\title{
APORTES CULTURALES DE LOS MORISCOS EN TÚNEZ
}

\author{
RAJA YASSINE BAHRI \\ Universidad de La Manouba (Túnez)
}

Fecha de recepción: noviembre 2008

Fecha de aceptación: marzo 2009

Un siglo de intento de asimilación de los moriscos no modificó para nada su personalidad. Éstos quedaron muy fieles a sus tradiciones, padeciendo una injusticia, al ser obligados a convertirse por la fuerza a la religión cristiana y como escapatoria intentaron consolidar aún más sus lazos con sus hermanos del otro lado del Mediterráneo. Felipe II comprendió dónde radicaba el mal y reforzó su política de asimilación de esa minoría. Se repitieron los decretos prohibiéndoles moverse de sus pueblos de origen, cambiar de domicilio o de señor, circular o acercarse a la costa, embarcarse hacia Berbería, comunicarse con los corsarios y relacionarse o encubrir a sus hermanos aragoneses y granadinos.

A pesar de todas las medidas tomadas al respecto, la segundad mitad del siglo XVI fue el escenario de una ida y vuelta continua a través del Mediterráneo entre España y Argel. El proceso de Abdallah Alicaxet es un caso ejemplar de los moriscos que se establecieron del otro lado del Mediterráneo en un momento en que las fronteras estaban totalmente cerradas ${ }^{1}$. Las denuncias a este respecto abundan. Los procesos inquisitoriales atestiguaban que, eventualmente, buena parte de los moriscos perseguidos mantenían una correspondencia fija con el «rey» de $\mathrm{Argel}^{2}$ y el de Francia. El peligro

1. A.H.N.: Inq. leg. 548, $\mathrm{n}^{\circ} 1$. Alicaxet era un corsario que participó en la organización y desarrollo de una conspiración para una «eventual» segunda revuelta en la Península. Había salido de España a los veinte años, en 1556, para instalarse en Berbería. Entabló gran amistad con el rey de Argel y participó en la batalla de Lepanto formando parte de la armada otomana. Alicaxet se dedicaba al corso cautivando cristianos que vendía en Argel, saqueando los barcos que encontraba y ayudando a los cristianos nuevos a pasar al otro lado del Mediterráneo en un momento en que les estaba prohibido dejar la Península.

2. Para más detalles sobre las relaciones de los moriscos y el imperio otomano véase: BORONAT Y BARRACHINA, Pascual: Los moriscos españoles y su expulsión, Valencia, 1901; CARDAILlAC, Louis: «Le Turc, suprême espoir des Morisques», Extrait du Cahier Série Histoire, Tunis, 1974, nº 1, Tome II, págs. 
de una eventual intervención del imperio otomano creaba una psicosis de peligro en España, una atmósfera de inseguridad, de duda y de perplejidad. Muchos procesos inquisitoriales testifican que muchos moriscos valencianos se habían unido al corsario magrebí y otomano y recorrían el mar Mediterráneo practicando el comercio de venta y compra de los cautivos cristianos; otros mantenían una correspondencia entre las aljamas y las potencias extranjeras y otros preparaban un levantamiento de los moriscos apoyados por los turcos. El país parecía muy amenazado y el morisco vuelve a ser un problema nacional e internacional.

Su rechazo de la conversión, su negación de la integración, de la asimilación, así como otros motivos políticos y religiosos llevaron al gobierno de Felipe III a decidir finalmente la expulsión. La decisión fue tomada el 9 de abril de 1609. Los moriscos tenían que ser expulsados de toda la Península Ibérica empezando por los de Valencia, considerados como los más islamizados y por consiguiente peligrosos. El bando de expulsión de los moriscos valencianos, dictado por el Marqués de Caracena el 22 de septiembre de 1609, prescribe algunas excepciones, ordenando que deben quedarse el 6 por ciento de las familias moriscas con objeto de atender ciertas labores relacionadas con el trabajo del campo, lo que denota que los moriscos expulsados eran especialistas en muchos oficios agrícolas. También pueden quedarse los menores de seis años con sus padres, aunque la madre sea cristiana nueva ${ }^{3}$. Se decretaron otros edictos dando permiso a los moriscos a llevar los hijos menores, con la única condición de pasar por un país cristiano para confirmar aún más su cristiandad. En 1610 se verificaba la presencia en España de 1.832 niños menores de 7 años. Pero muchos moriscos rehúsan abandonar sus tierras y se levantan en algunas zonas. Salieron en total, según la estimación de Henri Lapeyre, 275.000 moriscos del reino de España ${ }^{4}$. Los autores contemporáneos de los moriscos elevan la cifra a $340.672^{5}$ y no cesan de enumerar las crueldades infligidas a los desgraciados moriscos echados obligatoriamente de sus territorios. Según la información suministrada por Fonseca y Juan Luis de Rojas, muchos moriscos fueron asesinados ${ }^{6}$ en el camino de la expulsión o en la costa argelina. Asimismo según fuentes notariales estuvieron más expuestos al pillaje o murieron en la travesía por cansancio y hambre. No todos los países de destino fueron siempre

37-46. GARCÍA MARTÍNEZ, Sebastián: «Bandolerismo, piratería y control de moriscos en Valencia durante el reinado de Felipe II», Estudis, Valencia, 1972, 1, págs. 85-167; CARRASCO, Raphael: «Peril Ottoman et solidarité morisque (La tentative de soulèvement des Morisques des années 1577-1583)», Revue d'histoire Maghrébine, Tunis, 1982, n²5-26, págs. 33-50 ; TEMIMI, Abdeljelil: «Une lettre des Morisques de Grenade au Sultan Suleiman Al-Kanuni en 1541», Revue d Histoire Maghrébine, 1975, 3, págs. 99-106; VINCENT, Bernard: «Les bandits morisques en Andalousie au XVIè siècle», Revue d'Histoire Moderne et Contemporaine, Paris, Juillet-Septembre 1974, XXI, págs. 389-400

3. Danvila y Collado, Manuel: La expulsión de los moriscos españoles, Madrid, 1889, págs. 298-300.

4. LAPEYRE, Henri: Géographie de l'Espagne morisque, París, 1959, pág. 206.

5. Boronat y BARRACHINA, Pascual: Op. cit. págs. 304-305.

6. RoJAs, Juan Luis de: Relaciones de algunos sucesos prosteros de Berbería, Lisboa, 1612, f. 24-v; FONSECA, Damián: Justa expulsión de los moriscos de España, con la instrucción apostasia y traición dellos: y respuesta de las dudas que se ofrecieron acerca desta materia, Roma, 1612, págs.. 272 y ss. 
acogedores, la población indígena africana se reveló hostil a la inmigración española aunque se trataba de musulmanes.

El decreto de expulsión, promulgado tras 100 años de intentos de conversión sincera, pone de manifiesto que el morisco nunca había aceptado la integración en el mundo cristiano y que se había considerado siempre muy distinto de la población dominante. Mohamed Alguazir, morisco expulsado residente en Marruecos, señala que: «en más de cien años después de la conquista de España por los cristianos, jamás salieron de su ley» a pesar de que la Inquisición «cada día les quemaba por Moros». Los procesos de la Inquisición posteriores a la expulsión aclaran y ponen de manifiesto que el morisco una vez en Berbería consolidó aún más su identidad religiosa acudiendo a unas escuelas religiosas donde aprendían algunas oraciones, pues las autoridades regionales les ayudaban en este sentido.

De repente, el morisco cambia de medio geográfico y cultural y se encuentra en medio de una población musulmana entre la que estaba esparcida una antigua población de origen español ${ }^{7}$. En efecto, por la proximidad a la Península Ibérica, Argelia, Marruecos y Túnez venían recibiendo oleadas de andaluces desde fechas muy lejanas. El continuo vaivén de hombres de uno a otro lado del Mediterráneo se convirtió en vía de dirección única desde que el predominio cristiano se hizo aplastante. Con la reconquista, muchos mudéjares emigraron hacia Túnez y se establecieron en la capital. Algunas calles de la Medina hoy en día llevan su nombre: calle de Granada, calle de los Andalusíes y calle de Sevilla. Cada región, cada gran ciudad reconquistada significaba un nuevo éxodo que empobrecía al-Andalus y enriquecía las tierras norteafricanas, proporcionándoles artesanos, mercaderes, intelectuales y hombres de Estado. La mayor parte de esta emigración se dirigió a las ciudades y constituyó el núcleo de su burguesía.

La caída del reino de Granada le dio nuevo impulso a esta emigración, y durante todo el siglo XVI continuó el incesante éxodo de moriscos. A pesar de que se les prohibía acercarse al litoral o vivir cerca de la costa, particularmente de la costa valenciana, muchos moriscos lograron salir y pasaron a la otra orilla del Mediterráneo, ya por propia voluntad, ya aprovechando alguna incursión pirática. Las huidas desde los reinos de Granada y Valencia a Berbería durante el siglo XVI fueron numerosas. Pero la gran inmigración hacia el Magreb se llevó a cabo a partir de 1609 con la expulsión general.

Marruecos, por motivos de guerras y de disturbios internos, no estaba dispuesto a recibir a miles de inmigrantes andaluces, aunque a pesar de ello muchos arribaron allí y la mayoría de los moriscos que llegaron a Marruecos en 1610 eran andaluces, castellanos y extremeños, aunque no muy numerosos en comparación con los que llegaron a Argelia o Túnez. Llegaron allí unos 40.000 moriscos, vestidos a la española y hablando castellano. Estos no fueron bien acogidos, se les acusaban de cristianos y en ocasiones sufrieron martirios.

7. BRUnSCHVIG, Robert: La Berbérie orientale sous les Hafsides des origines à la fin du XVe siècle, tomo 2, Paris, 1947. 
Un gran número de moriscos, sobre todo valencianos, se dirigieron a las costas de Argel. Estos fueron los que tuvieron peor suerte pues encontraron tribus bárbaras que los despojaron y maltrataron sin piedad. Pero el grupo más compacto y el más afortunado fue el que arribó a tierras de Túnez. Como en el resto del Magreb, los recién llegados encontraron allí muchos andalusíes que habían emigrado cuando empezó la reconquista, pues había efectivamente andalusíes en Túnez por lo menos desde el siglo XIII. Muchos sevillanos llegaron allá después de la conquista de Sevilla en 1248. También llegaron muchos del Levante e Ibn Jaldún, que escribía en el siglo XIV, dice que entre ellos había ilustres familias de príncipes, sabios, poetas y guerreros. De nuevo se intensificó la corriente migratoria a partir de 1492, dirigiéndose a la capital de Túnez, Sfax, Gabes y Susa.

Pero la gran inmigración se llevo a cabo en 1609 y se la computa en cerca de ochenta mil personas, número considerable para un país pequeño y poco poblado ${ }^{8}$. Túnez en aquel momento tenía un millón de habitantes en total y la inmigración iba a aumentar la población del país de un 10 a un 15 por ciento. Entre ellos había pocos valencianos, siendo la mayoría castellanos y aragoneses. Éstos tuvieron la suerte de ser muy bien acogidos por el dey turco Uzmán, el cual comprendió que su aportación sería preciosa para el desarrollo del país ${ }^{9}$. Túnez en aquella época era un país primitivo, muy poco poblado a causa de las epidemias y guerras que saquearon y empobrecieron el país. Uzmán Dey sabía que la inmigración andalusí podía ser beneficiosa para un país despoblado y primitivo. Era un rey tolerante y prudente, de espíritu abierto y hasta cierto punto proclive al progreso. Sabía que la entrada a su país de gente de alta calidad cultural y de un nivel superior, podría mejorar el país y consolidar su posición frente a la Sublime Puerta, de la que era vasallo. Se organizó para recibir oleadas de emigrantes españoles de un nivel artesanal y agrícola superior e integrados por artesanos, comerciantes, agricultores, pequeños industriales, artesanos arquitectos y artistas, a los que concedió muchos privilegios desde el primer momento ${ }^{10}$. Dispensó de tasas portuarias a las naves que los traían, dio una moratoria fiscal a éstos para los primeros años de su instalación, les dio la posibilidad de escoger comarcas o tierras, les proporcionó semillas y trigo para sembrar la tierra y otras medidas de protección oficial para facilitar su instalación.

Según el testimonio de un morisco expulsado en Túnez, los moriscos fueron muy bien acogidos por el rey:

Alli, nos recibieron Uzman Dey, rey de Túnez, de condición soberbia y para nosotros manso cordero, Citi Bulgaiz con su santidad, y la gente con su Içlam; y todos procurando acomodarnos y regalándonos con grande amor y amistad. Uzman Dey quitó una costum-

\section{Ibidem.}

9. PIgnON, Jean: «Un document inédit sur la Tunisie au début du XVIIè siècle», Les Cahiers de Tunisie, Tunis, 1956, 15, págs. 301-326.

10. Epalza, M. DE-Petit, R.: Études sur les Morisques andalous en Tunisie, Madrid-Tunis, 1973, págs. 206-209. 
bre que abía de pagar cada bajel cien escudos por la entrada, fundando en que se animase a traernos a esta ciudad ${ }^{11}$.

Numerosas fueron las personas que apoyaron y obraron en favor de la inmigración morisca. Se puede citar a Abu al-Gayz al-Qassas, personaje religioso y de letras, quien obligó a los habitantes de Túnez a recibir en sus casas a los recién llegados, y puso a su disposición instructores en lengua árabe para que se les enseñasen la lengua y la religión musulmana. Del mismo modo abrió también algunas mezquitas y apoyó que la instrucción religiosa de los moriscos pudiera hacerse en castellano, si era ésta la única lengua que sabían. Abu al-Gayz al-Qassas se desvivió en favor de los moriscos e hizo cuanto pudo para que les fueran concedidos alojamientos dignos y alimentación. Por otro lado se les concedió una libertad completa de movimientos ${ }^{12}$. Distribuyó limosna a los desfavorecidos, pues muchos de ellos habían sido despojados de sus bienes en la travesía, aunque otros consiguieron llegar a Túnez con dinero y objetos personales de gran valor. Gracias a la protección de al-Qassas, los moriscos fueron desde los primeros momentos inmersos en un ambiente de hospitalidad y pudieron seguir usando la escritura en español y ello les permitió desarrollar una literatura propia en el exilio ${ }^{13}$.

Según los testimonios inquisitoriales, los moriscos que llegaron a Túnez eran en su mayoría cristianos, hablaban castellano y eran en buen número rubios con ojos azules. Desde los primeros momentos fueron obligados a convertirse al Islam y debían adoptar el modo de vida tunecino, algo que les resultó difícil. Se sentían totalmente diferentes y muy ajenos en su modo de vivir ${ }^{14}$. En el XVIII la separación era aún muy visible, y aún hoy, aunque amortiguadas, quedan huellas. Los moriscos más adinerados se quedaron en Túnez capital y encontraron refugio en la Medina, mientras que el resto de la población morisca se estableció en cuatro grandes zonas:

\section{Ibídem.}

12. KRESS, Hans-Joachim: «Elements structuraux «andalous» dans la genèse de la géographie culturelle de la Tunisie», Ibla, Tunis, 1980, 145, págs. 237-284..

13. BERnABÉ PONS, Luis F., «L'écrivain morisque hispano-tunisien Ibrahim Taybili (Introduction à une Littérature Morisque en Tunisie)», Mélanges d'Archéologie, d'Épigraphie et d'Histoire offerts à Slimane Mustapha Zbiss, Tunis, 2001, pp. 249-272.

14. A.H.N.: Inquisición, Leg. 548 Número 5 (1611) María Alaviat - número 21 (1624) Miguel Alias Amete - número 13 (1720m 20 de septiembre) Amet, esclavo.

Número 21 (1624) Miguel Boris (Amete).

Leg. 549 número 4 (1613) María Magdalena - número 13 Juan Borbay (1620).

Leg. 550 número 7 (1612) Jaime Carroz- número 29 Juan Domingo.

Número 21 (1621) Juan Chaez - número 24 (1816) Hamet Chocor.

Leg. 551 número 16 (1612) Francisco Felipe - número 8 (1624) Jerónimo Farrach - número 12 (1717)

Juan Bautista Ferrer.

Leg. 552 número 34 (1611) Proceso de María Tigir- número 22 (1612) Angela Malech- número 2 (1613) Angela Mariana- número 18 (1613) Jaime Cahat.

Leg. 553 Número 24 (1616-1621) Francisco Pérez (Ali)- número 27 caja 2 Gil Pérez (1582).

Leg. 555 número 17 (1621) Cristóbal Socaltre.

Leg. 556 número 12 (1621) Jerónimo Verge.

Leg. 844 número 23 (1614) Jerónimo Juana María. 
1-El valle del río Medjerda y en los alrededores de Túnez.

2-Llanuras septentrionales en el camino Túnez-Bizerta.

3-Llanuras septentrionales en el camino Túnez-Nabeul.

4-Zaghouan, junto a la montaña que da agua al acueducto de Túnez ${ }^{15}$.

Como decíamos, los más adinerados, los sabios, y miembros de corporaciones urbanas se instalaron en un barrio alto, cerca de la alcazaba y palacio del gobierno, alrededor de las actuales calles de los Andalusíes y plaza del Palacio. Se instalaron también en ciertos barrios de la capital, donde ya de antes existía una «calle de los Andalusíes» y un «zuqaq al-Andalus», en la Medina cerca de Bab Mnara y de la Kasba. Los artesanos ocuparon arrabales, especialmente al norte del recinto antiguo de la ciudad, en Halfawín (barrio de los esparteros), Bab-Suwayqa (puerta del zoquito, donde se construyó una mezquita que aún se conserva) y al-Fajjarín (plaza de los alfareros).

Los hortelanos y pequeños industriales se establecieron en las afueras de la capital en unos nuevos centros creados para ellos: Ariana, Djedaida, Teburba, etc., donde continuaron sus actividades tradicionales, incrementaron las pequeñas industrias textiles y favorecieron el comercio entre las regiones con la introducción del carro. Otros encontraron refugio en el Bardo y dotaron la ciudad de nuevos jardines en los que plantaron una gran variedad de arboles frutales. Se establecieron también en la Manuba, la Sukra, Cartago, Gammarth, Mornag, Sidi Bou Said y Radés.

Pero el grupo más numeroso estaba compuesto de labradores y agricultores y éstos se establecieron en las zonas agrícolas vacantes, en especial en el Cabo Bon (Grombalia, Solimán, Turki, Belli y Nianu), y en el valle del Medjerda. Otros poblaron regiones abandonadas en lugares elevados, o en ruinas de ciudades romanas como Testur, Medjaz-el Bab, Djeida, El Batán, Slughuia, Qalaat-el-Andalus, El Alia, Bizerta, Raf Raf, Ras El-DJebel, Porto Farina, Medjez El-Bab, Grish el-Oued, Menzel Burguiba, Menzel-Djemil, Metline, Mater, Aousdja y Tebursuk. Casi todos se establecieron en el norte del país o bien en las riberas del río más grande del país -el Medjerda- o bien en la región del Cabo Bon, región muy fecunda. Muchos escogieron la región montañosa de Zaghouan, muy abundante en agua subterránea -la que desde el tiempo de los romanos surtía a Cartago- ${ }^{16}$.

Desde su llegada a Túnez, los moriscos tendían al agrupamiento por entidades regionales de procedencia, es decir, que aún en tierra extraña el valenciano seguía sintiéndose distinto del granadino y del mudéjar castellano. La especialización profesional también imponía criterios de agrupación, conforme a la antigua costumbre de reunir mercaderes y artesanos en calles y barrios especiales. Tal ocurrió en la Medina de Túnez, o en Bizerta, que tenían cada una su Humat al-Andalus o barrio de los andalusíes, dotado de cierta autonomía.

15. EPAlZA, Míkel de: Los Moriscos antes y después de la expulsión, Madrid, 1992.

16. SERNA, Alfonso de la: Imágenes de Túnez, Madrid: Instituto de Cooperación con el Mundo Árabe, 1990, pág. 249. 
La prosperidad artesanal de Túnez, y en menor grado de otras poblaciones, recibió gran impulso con la llegada de estos refugiados, muchos de los cuales llegaban con su caja de herramientas. Mejoraron la técnica de los tejidos de seda en todas sus fases. Especial importancia cobró la fabricación de bonetes (chechía) cuyo vocabulario artesanal todavía hoy es de origen español en buena parte: «Abarcar», «afinar», «banku», «batitur», «kabisa», «kushnilla». Teyssier ${ }^{17}$ ha agrupado cuarenta y siete vocablos que se emplean hasta hoy en día en el zoco de la chechía en la Medina de Túnez. Muchos estudiosos se han ocupado en la fabricación de la chechia en Túnez como John D. Latham $^{18}$, Sophie Ferchiou ${ }^{19}$ o L. Valensi ${ }^{20}$, poniendo en valor la aportación morisca en su resurgimiento y desarrollo nuevo en el siglo XVII. La cerámica también tiene una tradición morisca en Túnez. La aportación morisca consistió en una renovación de los temas, tratados con brillante colorido. Los motivos andaluces también se hallan presentes en la decoración pictórica de techos, frisos, paredes y patios. La renovación arquitectónica que conoció la capital se manifestó en la construcción de casas suntuosas, algunas con patios de mármol, estucos y azulejos, fuentes y jardines de acusado ambiente andalusí. Jacques Revault hace una descripción de estos palacios ${ }^{21}$, citando los de Dar Haddad, Dar-Balma, Dar el-Asfuri, Dar Kastalli y tantos otros.

Las mejoras en el terreno agrícola fueron muy notables. Todos los que se han ocupado del país tunecino, historiadores, geógrafos y viajeros, han señalado el gran aporte vital que para él supuso la llegada de muchos miles de expertos campesinos que renovaron las técnicas de riego, plantaron árboles frutales y dieron un gran impulso a ciertos cultivos, especialmente la horticultura y la jardinería ${ }^{22}$. El aporte más destacado es la plantación de olivos y naranjos, plantaciones muy desarrolladas en España ${ }^{23}$. En efecto, las guerras en Túnez aceleraron la destrucción de todas las antiguas plantaciones romanas. Los moriscos aprovecharon estas tierras para dinamizarlas y dotarlas de nuevas plantaciones de origen español. De repente las regiones tomaron el aspecto de una auténtica comarca española. Los moriscos introdujeron nuevas técnicas productivas como la instalación de un sistema original de irrigación, las acequias, plantaciones lineales y más ordenadas, poda de los árboles e injertos, particularmente en el valle de

17. TEYSSIER, Paul: «Le vocabulaire d'origine espagnole dans l'industrie tunisienne de la chéchia», EPALZAPETIT: Op.cit., pág. 308.

18. LATHAM, John D: «Contribution à l'Étude de l'émigration andalouse et à sa place dans l'histoire de la Tunisie», Ibidem, p. 21.

19. FerChIOU, Sophie: Technique et société: la fabrication de la chéchia en Tunisie, Paris, 1971.

20. VALENSI, Lucette: «Islam et capitalisme: production et commerce des chéchias en Tunisie et en France auX XVIIIe et XIXe siècles», Revue d'histoire moderne et contemporaine, Paris, 1969, XVII, págs. 376400.

21. ReVault, Jacques: Palais et demeures de Tunis (XVI-XVIIe siècles), Paris, 1967.

22. VALENSI, Lucette: Fellahs tunisiens. L'économie rurale et la vie des campagnes aux 18è-19è siècles, Paris, 1977, págs. 103-104.

23. Hedi CHERIF, Mohamed: «Propriété des oliviers au Sahel des débuts du XVIIè à ceux du XIXè siècle», Actes du $1^{\text {er }}$ Congrès d'histoire et de civilisation du Maghreb, Tunis, 1978, págs. 209-252. 
la Medjerda y en Cap Bon. Por otro lado se introdujo un sistema de irrigación específico para transportar el agua de las pendientes vecinas hasta los pequeños aguaduchos que desembocaban en las plantaciones. En Soliman cavaron pozos para la irrigación de los olivares alejados de los ríos. Allí se daban excelentes cosechas. En el siglo XVIII todavía los pueblos de origen español eran florecientes, aunque hoy están decaídos y la huella hispánica tiende a borrarse. Sigue siendo fuerte en algunos como Testur, situado en el valle del Medjerda, que, teniendo las tierras más fértiles de Túnez, se encontraba a la llegada de los moriscos entregado al pastoreo y, a lo sumo, al cultivo extensivo de cereales. Ellos introdujeron cultivos irrigados en las llanuras bajas, leguminosas y cereales en las tierras de secano situadas a media altitud, reservando las más altas para el bosque.

La conciencia de su individualidad y de su superioridad les permitió mantener relativamente pura la etnia y el folklore del grupo durante largo tiempo ${ }^{24}$. Los moriscos que llegaron después de 1609 estaban más hispanizados y fueron difíciles de integrar en una sociedad tunecina primitiva y desvertebrada en sus aspectos urbanos. La endogamia estaba entre ellos muy generalizada: un viajero francés relata que las mujeres moriscas preferían quedarse solteras antes que casarse con un beduino. Eran trabajadoras, astutas, inteligentes y buenas amas de casa. Eran conscientes de que pertenecían a un mundo superior y más civilizado. Según el testimonio de Juan de Terpas, quien encargó a un amigo el presentarle una mujer morisca antes de su traslado a Argel porque piensa que las mujeres aragonesas eran más hábiles en las tareas domésticas que las argelinas, «le tiene encargado la busqueda de una mujer morisca para pasarla a Argel para que le sirva y casarse con ella porque las de allá no aciertan a servir ni guisar como las de acá» ${ }^{25}$.

Igualmente las moriscas revolucionaron la tradición vestimentaria tunecina de la época. Aznar de Cardona, incansable detractor de los moriscos hizo una descripción muy detallada en 1612 de la mujer morisca en el momento de la expulsión y dice lo siguiente:

Con sus joyas, grandes medallones de plata que descansaban sobre su pecho, colgadas de su cuello, con cadenas, collares, pendientes, pulseras, corales, con mil mezcolanzas y mil colores en sus vestidos, como si hubiesen querido disimular algo de los sufrimientos de su corazon ${ }^{26}$.

Y más adelante añade;

...pues, cada una lleva más oro que otros tienen de caudal en las tiendas más ricas, y es de suerte, que las más mínimas se adornan con cosas que las reinas de esta tierra no lleva-

24. DaChraOUI, Farhat, «A propos de la réalité culturelle des Morisques en Tunisie», in L'Écho de la prise de Grenade dans la culture européenne aux XVIè et XVIIè siècles, in Actes du Colloque de Tunis, Tunis, 1994, pág. 60.

25. A.H.N.: Inq. Libro 964, f. 359.

26. BAHRI, Raja: «Errance des Morisques en Méditerranée, après 1'expulsion de 1609», L 'Afrique au XVII siécle, Mythes et réalité, Actes du VIIè colloque du Centre International de Rencontres sur le XVIIè siécle, Tübingen, 2003, pp. 189-201. 
ban antes de nuestra venida y no han dejado estas cosas de hazernos daños, pues la invidia es un benenoso animal ${ }^{27}$.

Hoy en día muchas costumbres de la mujer tunecina son originarias de la época morisca. Las más acomodadas suelen tener criados para ocuparse de las tareas domésticas y de los niños y ellas pasan el tiempo cumpliendo obligaciones sociales, reuniones, visitas, ceremonias familiares o bien suelen pasar unas tardes en el baño, lavándose y charlando con amigas. Son igualmente buenas esposas y la mayoría del tiempo están sometidas al marido ${ }^{28}$. El viernes por la tarde suelen ir a la mezquita o a la zawiyya a pasar unos momentos con algunas amigas acompañadas de sus hijos cuando son pequeños.

Otras se dedican a ciertos trabajos manuales, hacen punto todo el día, bordan o hilan. En muchas regiones y particularmente en el Alia y Qalaat-el-Andalus las mujeres siguen con el vestido morisco primitivo que consiste en un traje largo de color llamativo con mangas anchas, preferiblemente amarillo, verde o azul ${ }^{29}$. Casi todas salen a la calle con la almalafa tejida de lana, muy similar a la que aparece en los grabados contemporáneos de moriscas de Weiditz o Heylan. Usan los antiguos productos naturales a base de plantas para pintarse de la misma manera que las moriscas (gustan, por ejemplo, de llevar lunares). En cuanto a la joyería, en Túnez las mujeres llevan todavía las arracadas en las orejas y pulseras a la morisca (Chnachen).

En cuanto al arte culinario, casi toda la comida tunecina actual saca su origen de la época morisca ${ }^{30}$. Un buen número de platos datan del siglo XVII. Los moriscos introdujeron los platos agridulces a base de frutas, nueces y almendras. Muchos platos son preparados a base de harina, sémola y de carne. Hoy en día en Túnez capital, preparan, «basabán», (mazapán), «cunfit» (confite), «menteque» (manteca), «limún», (limón), «kullares» (collares de longaniza de cordero), la «oja», la (olla). En la cocina de procedencia andaluza entran platos de carne condimentados con abundantes especias y confituras hechas a base de harina miel o azúcar. Otras recetas son totalmente andaluzas como las empanadas (banadj). El tema de la gastronomía «morisca» merecería un estudio detallado, pues muchos platos tienen un discutido origen andalusí o morisco, particularmente en la capital del país.

Todavía hoy, físicamente, el tunecino de ascendencia andalusí se distingue del indígena por los rasgos de su fisionomía y el color claro de su tez y demuestra un cierto orgullo por su origen andaluz. El uso del español y del valenciano ya se ha perdido, pero quedan infinidad de detalles materiales, de costumbres y nombres de familias que recuerdan su origen; como por ejemplo la onomástica, con la familia Chebil de Sevilla,

27. Ibid. p. 201

28. Fournel-Guerin, Jacqueline: «La femme morisque en Aragon», in Les Morisques et leur temps, Paris: ed. CNRS, 1983, pág. 525.

29. Sugier, Clémence: « Parures traditionnelles des Tunisiennes comparées à celles des femmes de l'Islam espagnol»: Études sur les Morisques andalous, Tunis, 1983, págs. 179-193.

30. Harzallah, Fatima: «Les femmes tunisiennes et l'influence andalouse», in L'Écho de la prise de Grenade dans la culture européenne aux XVIè et XVIIè siècles, Tunis, 1994. 
Merichcou de Morisco, Kortbi de Córdoba, Gharnati de Granada, Balanzi de Valencia y Saracosti de Zaragoza, Zbiss (Llopis), Zafrán (azafrán), Xátiva de Játiva, Tage (Tajo), Taruel (Teruel), Soria, Sordu de Sordo, Sancho, Zaragusti, Rachico, Ricardun, Cabadu, Pérez, Pintur, Negro, Al-Munakabi (Almuñécar), Merquicu, Merichco, Méndez, Menara, Medina, Marcu, Manacho, Malaki, Kuinka, Kristu, Jhaín (Jaén), Ichbili, (Sevilla), Huiski (Huesca), Herrera, Harrús, Harabún, Garnata (Granada), García, Galantu, Cortubi (Córdoba), Castali (Castilla), Caravaca, Cantalán, (catalán), Buguerra, Blanco, Betis, Benavides, Bekil, Barin, Bantur, Balma (Palma), Arruich, (Ruiz), Andulsi, (andalusí), Alicanti, (Alicante). etc...

En algunas regiones fundadas por los moriscos, las fachadas de ladrillo de sus casas recuerdan al mudéjar aragonés, y los remates cónicos de los minaretes las torres de Toledo y Burgos. En Testour, una de las peculiaridades más curiosas de sus casas consiste en que hay puertas adornadas con clavos que forman dibujos cruciformes. Esos mismos dibujos figuran en algunas casas de la Medina de Túnez para diferenciar al cristiano nuevo emigrado de los indígenas del país.

Algunas de sus costumbres pasaron al resto de los habitantes. Por ejemplo los palos de la baraja tunecina se llaman dinar (oros), bastun (bastos), esbata (espada) y kub (copas). Entre los millares de documentos jurídico-comerciales de los archivos del consulado de Francia en Túnez, cuya fecha inicial es el año 1582, Míkel de Epalza ha encontrado 246 referentes a moriscos, ilustrativos de sus actividades profesionales y también de su nivel de instrucción, pues se comprueba que su grado de alfabetización era superior al de los demás musulmanes. Algunos firmaban con caracteres latinos muchos años después de la expulsión. Los moriscos revolucionaron la vida económica tunecina y estuvieron en el origen de un gran florecimiento económico y cultural ${ }^{31}$.

Su época brillante en Túnez parece llegar hasta 1650. La segunda mitad del siglo es de estancamiento: las autoridades no les eran tan propicias, los impuestos llegaron a ser excesivos. En el siglo XVIII el grupo ya estaba amenazado de disgregación y asimilación, excepto en ciertos lugares donde formaban mayorías compactas como en la localidad de Testur. En el exilio, los moriscos, aunque inmersos en un medio arábigo-musulmán, continuaron escribiendo literatura española, porque la mayoría de ellos hablaban español y no eran capaces de escribir en árabe con soltura. Oliver Asín cita, entre otros escritores, a Abd-al-Karim ben Ali Pérez, que en 1615 escribió una apología del Islam, incluyendo una diatriba, muy comprensible, contra la Inquisición y sus miembros; al morisco toledano Juan Pérez, que al establecerse en Testur tomó el nombre de Ibrahim Taibili y escribió poesía renacentista al estilo de Castilla. Un curioso pasaje de este escritor parece demostrar que conoció el Quijote en una edición hoy perdida. Se conservan también no pocas obras anónimas, la mayoría de contenido religioso, pero algunas inspiradas en la literatura castellana y así circulaban romances y poesías de Garcilaso, Góngora y Lope. Según Oliver Asín, Túnez fue la capital

31. HEDI CHERIF, Mohamed: «L'impact de la chute de Grenade», in L'Écho de la prise de Grenade dans la culture européenne aux XVIè et XVIIè siècles, Actes du Colloque de Tunis, Tunis: CERES, 1994, pág. 20. 
intelectual de todos los moriscos del Magreb. Allí se leían obras procedentes de Argel, como las poesías de Ibrahim de Bolfad, y de Marruecos, como la «Apología contra la ley cristiana» de Muhammad Alguazir, morisco de Pastrana. Esta supremacía literaria de Túnez refleja, sin duda, la mejor acogida y más favorables condiciones de establecimiento que encontraron los moriscos que allí llegaron. Hacia 1720 un viajero definía a los descendientes de los moriscos como «más civiles y corteses que los demás habitantes», aunque «arrogantes, severos y ávidos de gloria». Parece que todavía se hacen a cuenta de esto, chistes.

En conclusión, los moriscos fueron los constructores de un nuevo mundo en la otra orilla del Mediterráneo. Incrementaron la población tunecina con sucesivas oleadas de inmigración, cambiaron el panorama cultural con el aporte de una nueva literatura en español escrita en el exilio. Mejoraron el campo con la introducción de nuevas especíes de plantaciones, favorecieron el incremento de las pequeñas industrias y dinamizaron el comercio entre las distintas regiones; actividad muy desarrollada en España antes de la expulsión. 